\title{
Assessing the Influence of Anteroposterior Lip Position Based on Esthetic Line on the Perceived Attractiveness
}

\author{
Ha-Yoon Jung, Je-Seok Oh, Zheng Hui, Kwang Chung, Seunggon Jung, \\ Hong-Ju Park, Hee-Kyun Oh, Min-Suk Kook
}

Department of Oral and Maxillofacial Surgery, School of Dentistry, Chonnam National University, Gwangju, Korea

Purpose: The purpose of this study was to assess the effects of lip anteroposterior position based on esthetic line on the perceived attractiveness.

Materials and Methods: We selected a 20s female standard lateral photograph which was within average range of cephalometric analysis, modified lips anteroposterior position based on esthetic line into 5 pictures. This study investigated and compared the preference of facial profile among the groups; male : female and dental relevance: non-dental relevance. Total 255 judges (male : female $=138: 117$, relevant : non-relevant=159: 96 ) who were 20s to 30s were asked to rate these photographs based in lip attractiveness using visual analogue scale (VAS).

Result: All groups had similarity the average of VAS of moved backward lips $2 \mathrm{~mm}$ were highest and moved forward lips $4 \mathrm{~mm}$ were lowest. Comparing between male group and female group, there were significant differences in all pictures except for original which was not modified. In the dental groups, moved forward lips $2 \mathrm{~mm}$ had significant difference and the average in dental relevant group were lower than non-relevant group in lip protrusion.

Conclusion: The preference about lip protrusion was similar irrespective of dental knowledge or gender. All groups preferred retrusion of lips to protrusion of lips. In female group, they had higher the average of VAS. In relevant group, they disliked protrusion rather than retrusion of lips significantly.

Key Words: Aesthetic line; Anteroposterior lip position; Attractiveness

Corresponding Author: Min-Suk Kook

Department of Oral and Maxillofacial Surgery, School of Dentistry, Chonnam National University, 77 Yongbong-ro, Buk-gu, Gwangju 500-757, Korea

TEL : +82-62-530-5610, FAX : +82-62-228-8712, E-mail : mskook2@gmail.com

Received for publication November 29, 2013; Returned after revision December 18, 2013; Accepted for publication December 21, 2013 Copyright (C) 2013 by Korean Academy of Dental Science

(C) This is an open access article distributed under the terms of the Creative Commons Attribution Non-Commercial License (http://creativecommons.org/licenses/ by-nc/3.0) which permits unrestricted non-commercial use, distribution, and reproduction in any medium, provided the original work is properly cited. 
Introduction

Modern society has greater importance on appearance, and the face of an individual is a key factor in recognition of one's charm. Several factors such as shape of eyes, color of pupils, appearance of nose and lips, and so on in their harmony grants the esthetics to the individual.

According to recent studies, the main reason for adult orthodontic treatments is not structural or functional correction but improvement of facial appearance $^{1)}$. Moreover, the desires for more esthetic face are becoming more concrete with developments of orthognathic surgery, and those count as important factors in treatment plans ${ }^{2}$. The focus was on analyzing hard tissues on orthodontic diagnosis and treatment plan in the past, but recent processes focus on analyses of soft tissues in order to express improvements in facial esthetics ${ }^{3)}$.

Influential factors on lateral attractiveness include shape and height of nose, shape and protrusion of lips and forehead, shape and location of chin, thickness of soft tissue, skeletal and dental structure, and vertical height of face. Among those, variations in extent of protrusion of nose, chin, and lips have great influence ${ }^{4,5}$. Standard lines of lower facial soft tissue for analysis of relationships of nose, chin, and lips are E-line by Ricketts ${ }^{6}$, S-line by Steiner ${ }^{7}$, B-line by Burstone ${ }^{8)}$, and $\mathrm{H}$-line by Holdaway ${ }^{9)}$. $\mathrm{Hsu}^{10)}$ report that E-line by Ricketts ${ }^{6}$ has convenience with reliance clinically according to his study on consistency and dependability of mentioned standard lines.

Previous studies on preference of lateral attraction mainly were related to analyses on silhouette or extra-oral pictures with visual analogue scale (VAS) and ranking scale, and various results were driven with influential factors such as gender, age, ethnics, culture, era, level of professional knowledge, and so on. Foster ${ }^{4)}$ said that most assessments were in agreement in favorable lateral attraction in range of one to two millimeters but young subjects, female subjects, and orthodontists had tendency toward favoring protruded lips in lateral view, based his study on preference of lateral attraction with $2 \mathrm{~mm}$ deviation of lip protrusion on lateral silhouette of eighteen year old Caucasian woman with esthetic lines. Czarnecki et al. ${ }^{5}$ ) reported that evaluation of lip protrusion is influenced by protrusion tendencies of nose and chin and there is difference among genders on preference in their study on preference in lateral attraction via lateral silhouette maneuvered with mean esthetic values and alteration in protrusion of lips, nose, and chin. According to Farrow et al. ${ }^{11)}$ in their study on lateral attraction preference of Caucasian, African Americans, general dentists, and orthodontists on lip protrusion alteration with computer manipulation of a dark-skinned subject, said that no difference existed depending on their race, gender, and age. In the study of Mantzikos ${ }^{12)}$, he reported that no significant preferential difference was found in the study of lateral attraction for photos of Japanese woman age of twenty years with manipulated lip protrusion and mandibular location among Japanese Americans.

In Korea, Song and $\mathrm{Choi}^{13)}$ reported there was a significant difference in preference of lateral appearance depending on their gender after surveying preferential tendency of Korean university students on facial attractiveness, using lateral silhouette. Ko et al. ${ }^{14)}$, in the study of influence of gender and culture on standard for lateral appearance in Koreans in their 20s living in Korea or the United States, reported that no difference was found between genders, but they said that there according to cultural background, Korea and America, existed difference, stating that culture has greater influence. Regarding previous studies, researches on preference in lateral attraction vary depending on gender, age, orthodontic knowledge, and culture. In analysis of later facial appearance, there is a significant difference in preference between Korean and westerner. 
However, there are only few studies on preference in lateral facial appearance, and most of them used ordinal scales such as ranking scale for evaluation. In Korea, there is few of studies on preference in lateral facial appearance using continuous scale such as VAS. Therefore, the purpose of this study is to analyze difference in esthetic preference between professional group and public group by suggesting lateral facial pictures with modified anteroposterior position of lips using VAS, and compared the difference in preference between groups.

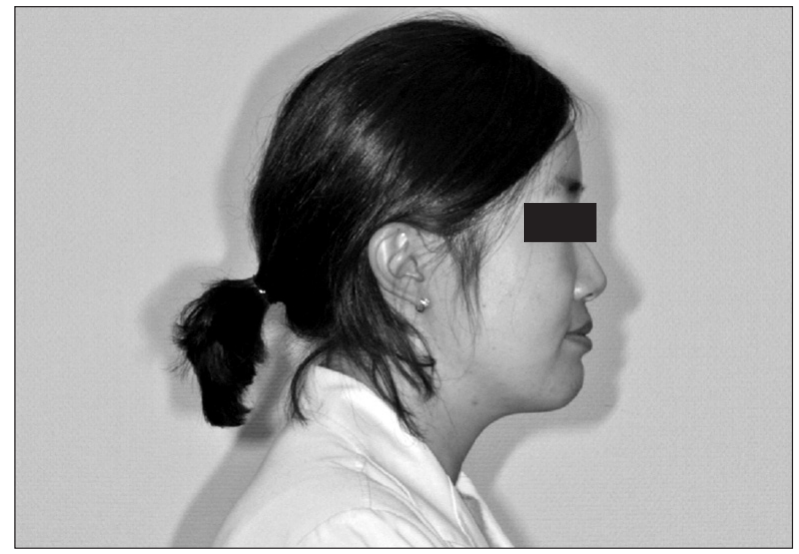

Fig. 1. Standard lateral photograph.

\section{Materials and Methods}

\section{Selection of Standard Lateral Photograph}

A lateral photograph of the in her 20s woman whose lip tip distance from her facial aesthetic line positioned within Korean normal range on cephalometric analysis was selected as the reference picture (Fig. 1). The model had a upper lip tip located in the $2.5 \mathrm{~mm}$ posterior to her facial aesthetic line and a lower lip tip located in the 1.0 $\mathrm{mm}$ anterior to it. It belongs to the normal range.

This study was researched in Department of Oral and Maxillofacial Surgery, School of Dentistry, Chonnam National University for 1 month. The model agreed this study and publication.

\section{Lateral Photo Sample Manipulation for a Eva- luation}

Using the photo editing software (Adobe Photo-

Table 1. Modification of lip position on the lateral facial photograph

\begin{tabular}{cl}
\hline Slide & \multicolumn{1}{c}{ Modification } \\
\hline Picture 1 & Retrusion of lips by $4 \mathrm{~mm}$ \\
Picture 2 & Retrusion of lips by $2 \mathrm{~mm}$ \\
Picture 3 & Original \\
Picture 4 & Protrusion of lips by $2 \mathrm{~mm}$ \\
Picture 5 & Protrusion of lips by $4 \mathrm{~mm}$ \\
\hline
\end{tabular}
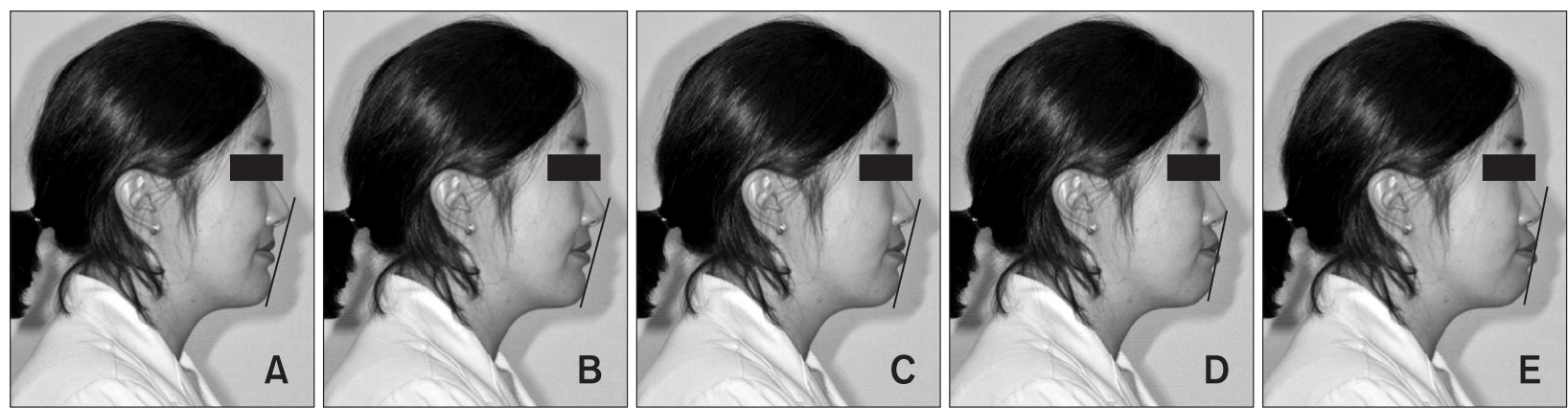

Fig. 2. (A) Modification of lip position on lateral facial photograph (Picture 1, $4 \mathrm{~mm}$ retrusion of lips from original photo). (B) Modification of lip position on lateral facial photograph (Picture 2, $2 \mathrm{~mm}$ retrusion of lips from original photo). (C) Standard lateral photograph (Picture 3, original photo). (D) Modification of lip position on lateral facial photograph (Picture 4, 2 mm protrusion of lips from original photo). (E) Modification of lip position on lateral facial photograph (Picture 5, $4 \mathrm{~mm}$ protrusion of lips from original photo). 
shop CS6 Extended; Adobe Systems, San Jose, CA, USA) with the selected standard lateral photograph, just anterior and posterior lip tip distance from the fixed facial aesthetic line were edited (Fig. 2). The lip tips of Picures 1, 2, 3, 4, and 5 were manipulated as much as posterior $4 \mathrm{~mm}$ (retrusion), posterior $2 \mathrm{~mm}$ (retrusion), $0 \mathrm{~mm}$ (origin), anterior $2 \mathrm{~mm}$ (protrusion), and anterior $4 \mathrm{~mm}$ (protrusion) from original positions respectively (Table 1, Fig. 2).

As a result the upper and lower lip tips of Picture 1 keep distances as much as posterior $6.5 \mathrm{~mm}, 4.0$ $\mathrm{mm}$ from the facial aesthetic line respectively, that means the both is posteriorly positioned beyond the Korean normal range. In case of Picture 2 the upper and lower lip tips keep distances as much as posterior $4.5 \mathrm{~mm}, 1.0 \mathrm{~mm}$ from the facial aesthetic line respectively, the upper is posteriorly positioned beyond the Korean normal range and the lower is positioned in the Korean normal range. Because Picture 3 is standard picture, the upper and lower lip tips belongs in the Korean normal range. In case of Picture 4, the upper and lower lip tips keep distances as much as posterior $0.5 \mathrm{~mm}$, anterior 1.0 $\mathrm{mm}$ from the facial aesthetic line respectively, and the both belongs in the Korean normal range. The upper and lower lip tips keep distances as much as anterior $1.5 \mathrm{~mm}, 5.0 \mathrm{~mm}$ from the facial aesthetic line respectively, only the lower is anteriorly positioned beyond the Korean normal range.

Table 2. Description of subjects

\begin{tabular}{ccc}
\hline Sex & Dental group $(n)$ & Ordinary group $(n)$ \\
\hline Male & 85 & 53 \\
Female & 74 & 43 \\
Total & 159 & 96 \\
\hline
\end{tabular}

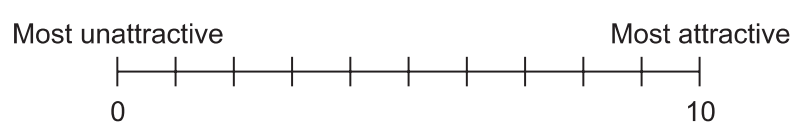

\section{Selection of Assessment Group}

This study is about the protruding lip on the attractiveness of the face and in order to evaluate the impact of it, total of 255 people (138 men, 117 women) belonging 20 s to 30 s were investigated by the survey. The comparison evaluation was going about not only gender differences in the preference, but also the group differences between the 159 dentistry worker who acquired dental knowledge for more 1 year and the other 96 people (Table 2).

\section{Evaluation Methods}

The VAS method has been introduced for the subjective preference evaluation in this study (Fig. 3). Gender of respondents and whether the dental knowledge acquisition was to determine through a questionnaire. Without any information about the manipulation of the picture and the facial aesthetic line, respondents rated aesthetic preference. Scoring sheet with $10 \mathrm{~cm}$ VAS that has the left end means "most unattractive (0)" and the right end means "most attractive (10)" was used after pretraining. Every scoring was determined subjectively by each personal judgement.

\section{Data Collection and Statistical Evaluation}

Finally after all data collected we measured the $10 \mathrm{~cm}$ VAS using a ruler refering to the Kazanis's evaluating method ${ }^{15)}$ using VAS and the result is recorded in a form of Excel spread sheet (Microsoft, Redmond, WA, USA).

Every statistical analysis was executed by SPSS Statistics version 17.0 (IBM Corp, Armonk, NY, USA) software. The means and standard deviations were calculated. And nonparametric ANOVA methods was introduced to confirm the significance of the preference differences between the groups. And Mann Whitney test was also executed to compare the preference of each of the groups.

Fig. 3. Scoring sheet with visual analogue scale. 


\section{Result}

To assess the preference of the male group, female group, dental professional group and public group according to the lip position anteroposteriorly based on the esthetic line, the mean VAS value of all groups was calculated, and nonparametric ANOVA was performed (Table 3 ). In all groups, the average score on the photograph not modified was close to 5. The average VAS scores of the Picture 1 and Picture 2 in which the lip was retruded, compared to the esthetic line were higher than that of Picture 3 , and the average VAS scores of the Picture 4 and Picture 5, in which the lip was protruded, were smaller. In addition, there was a statistically significant difference in preference on the rest of the photographs except for the Picture 3 which was not modified to each lateral photograph $(\mathrm{P}<0.05)$.

\section{Gender Preference}

The preference to the lip protrusion in the male

Table 3. Descriptive statistics according to preference

\begin{tabular}{cccccc}
\hline \multirow{2}{*}{ Statistic } & \multicolumn{5}{c}{ No. of picture } \\
\cline { 2 - 6 } & 1 & 2 & 3 & 4 & 5 \\
\hline Total average VAS & 6.04 & 6.97 & 5.19 & 2.95 & 1.96 \\
P-value & $0.040^{*}$ & $0.002^{*}$ & 0.529 & $0.018^{*}$ & $0.022^{*}$ \\
\hline
\end{tabular}

VAS: visual analogue scale.

${ }^{*} \mathrm{P}<0.05$

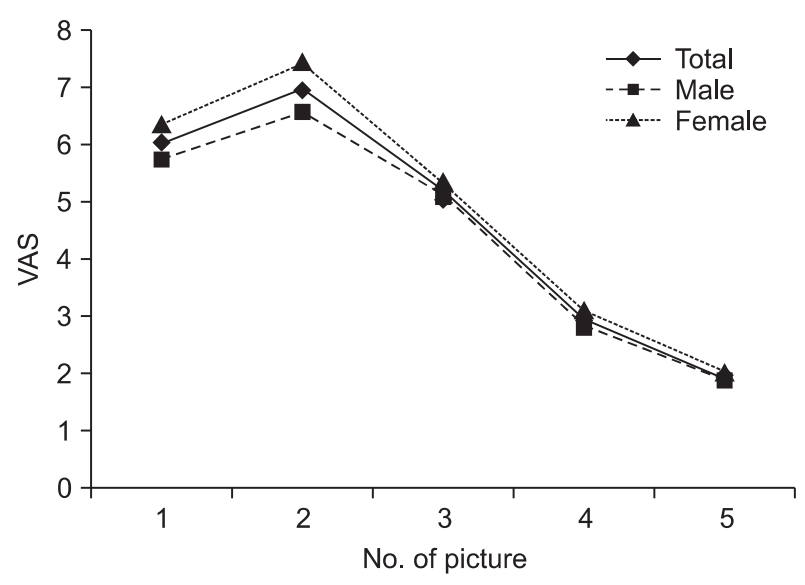

Fig. 4. Measured mean values of visual analogue scale (VAS) in gender. group and female group was confirmed by the average VAS value. Both groups favored the Picture 2 in which lip was retruded $2 \mathrm{~mm}$ from that of the original photograph. The photograph which was chosen to the most non-esthetic was Picture 5 in which lip was protruded $4 \mathrm{~mm}$ anteriorly. Unmodified Picture 3 was close to ' 5 '; near the middle value in both male and female groups. The average VAS values of Picture 1 and Picture 2, in which lip was retruded posteriorly, were higher compare to the unmodified photograph and Picture 4 and Picture 5 in which lip was protruded had smaller values (Fig. 4).

To compare the gender preference on the lip protrusion, the statistical analysis was performed using SPSS software. For the normality test, Shapiro-Wilk test was performed but did not follow a normal distribution, so the reliability verification was performed using Mann-Whitney test and measuring the test statistics (Table 4). The average VAS value of the female group was higher than all other evaluation groups, and in the results of the Mann-Whitney test, there was a statistically significant difference to all photographs except for the Picture $3(\mathrm{P}<0.05)$.

\section{Public Group and Dental Professional Group Preference}

The preference tendency of the community workers who acquired the dental professional knowledges at least 1 year and public group was analyzed. To compare the preference of each

Table 4. Descriptive statistics according to preference by gender

\begin{tabular}{cccc}
\hline Slide & Male & Female & P-value \\
\hline Picture 1 & $5.76 \pm 1.92$ & $6.38 \pm 1.96$ & $0.015^{*}$ \\
Picture 2 & $6.59 \pm 1.96$ & $7.43 \pm 1.75$ & $0.001^{*}$ \\
Picture 3 & $5.10 \pm 1.93$ & $5.29 \pm 1.75$ & 0.261 \\
Picture 4 & $2.82 \pm 1.59$ & $3.10 \pm 1.38$ & $0.023^{\star}$ \\
Picture 5 & $1.89 \pm 1.56$ & $2.03 \pm 1.22$ & $0.035^{*}$ \\
\hline
\end{tabular}

Values are presented as mean \pm standard deviation. ${ }^{*} \mathrm{P}<0.05$. 


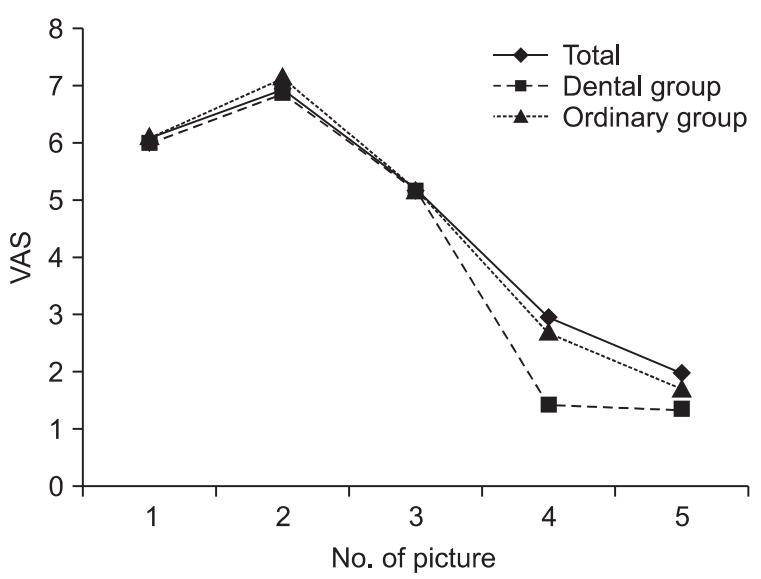

Fig. 5. Measured mean values of visual analogue scale (VAS) in dental relevance.

groups, the graph was made. Both group most preferred the Picture 2 in which lip was retruded $2 \mathrm{~mm}$ posteriorly based on the esthetic line in the original photograph. The photograph which was chosen to the most non-esthetic was Picture 5 in which lip was protruded $4 \mathrm{~mm}$ anteriorly. Unmodified Picture 3 was close to ' 5 '; near the middle value in the both dental professional and public groups. The average VAS value of Picture 1 and Picture 2 in which lip was retruded posteriorly was higher compare to the unmodified photograph and Picture 4 and Picture 5 in which lip was protruded have a smaller value (Fig. 5).

To compare the professional group and public group, the statistical analysis was performed using SPSS software. For the normality test, ShapiroWilk test was performed but did not follow a normal distribution, so the reliability verification was performed using Mann-Whitney test and measuring the test statistic (Table 5). In the results of the Mann-Whitney test, there was not a statistically significant difference of the preference tendency between dental professional group and public group when the lip was moved anteroposteriorly based on the esthetic line except for the Picture 4 in which lip was protruded $2 \mathrm{~mm}$ anteriorly, but in the case of Picture 4, there was a statistically significant preference difference $(\mathrm{P}<0.05)$. In the
Table 5. Descriptive statistics according to preference by gender

\begin{tabular}{cccc}
\hline Slide & Relevant & Not relevant & P-value \\
\hline Picture 1 & $6.01 \pm 1.85$ & $6.10 \pm 2.13$ & 0.757 \\
Picture 2 & $6.87 \pm 1.81$ & $7.15 \pm 2.06$ & 0.144 \\
Picture 3 & $5.20 \pm 1.81$ & $5.17 \pm 1.93$ & 0.919 \\
Picture 4 & $1.40 \pm 1.59$ & $2.67 \pm 1.30$ & $0.035^{\star}$ \\
Picture 5 & $1.33 \pm 1.64$ & $1.69 \pm 0.86$ & 0.095 \\
\hline
\end{tabular}

Values are presented as mean \pm standard deviation. ${ }^{*} \mathrm{P}<0.05$.

dental professional group, there was a evident nonpreferred tendency to the protruded lip based on the esthetic line.

\section{Discussion}

The standard lateral photograph chosen in this study had normal range of lip position anteroposteriorly in a relation to the esthetic line upon cephalometric analysis. Previous studies on preference of lateral attraction mainly were related to analyses on silhouette or extraoral pictures, and recent researches are concerned mostly with extraoral photos. A positive aspect of researches utilizing silhouettes is that influential factors on recognition of analyzer, such as eyes, head, ears, and so on, can be eliminated while silhouette can be recognized as a simple photo, not as a human head $^{11)}$. Regarding this, several methods like covering eyes or ears are used to eliminate such factors influencing analysis, but this study assumes that such factors had been incapacitated as only one factor, protrusion of lip, differed without change in other areas in single subject. Study by Choi and Lee $^{16)}$ on preference in lateral appearance also evaluated preference on lateral appearance of one subject without covering eyes.

On setting differential value during designing picture for lateral analysis with standard picture, previous studies did not present a concrete method to evaluate preference clearly but mostly have set random values. Burcal et al. ${ }^{17)}$ said alternation less 
than $4.0 \mathrm{~mm}$ on lateral aspect of face is difficult to recognize, but in all groups in study by Kazanis ${ }^{15}$ and this study, distance change less than $2 \mathrm{~mm}$ were recognized. Further studies need to have more stages with minute changes in order to identify the minimum value for esthetic recognition of facial change.

Faure et al. ${ }^{18)}$ suggested VAS, ratio scale, ranking scale, and so on as methods to evaluate preference on lateral appearance, and most preferential researches were involved with ranking scale. Phillips et al. ${ }^{19)}$, however, insisted that ranking scale causes overlaps with the same score and cannot collect consecutive data since it is an order based method. VAS is known as a quick, convenient, and reliable method in evaluation of facial esthetics ${ }^{15,17-20)}$. VAS method was utilized in this study to acquire objective appraisal on each picture, but several factors need to be considered in evaluation of objective opinions as in a facial esthetics using VAS. Firstly, it is difficult to assure that public group and dental professional group agree on the extremities of the scale, "most attractive" and "most unattractive." Also, it is hard to confirm that the same score from different members of the group represent the uniform value for specific facial appearance $^{21)}$.

Possibly influential factor in preference evaluation is gender, age, culture, ethnics, and so on. In study on preference of Caucasians, African Americans, general dentists, and orthodontists in lateral appearance on differentiating lip protrusion of a colored subject by Farrow et al. ${ }^{11)}$, no significant difference in preference was reported. Mantzikos ${ }^{12)}$ reported that there was no difference in preference of Japanese Americans according to age or gender on lateral appearance of Japanese woman in her 20s with variations in lip protrusion and anteroposterior position of mandible. Yet, whether these factors have influence or not is still in discussion ${ }^{21-25)}$.

In Korea, Song and $\mathrm{Choi}^{13)}$ reported there was a significant difference in preference of lateral appearance depending on their gender after surveying preferential tendency of Korean university students on facial attractiveness, using lateral silhouette. Ko et al. ${ }^{14)}$, in the study of influence of gender and culture on standard for lateral appearance in Koreans in their 20s living in Korea or the United States, reported that no difference was found between genders, but they said that there according to cultural background, Korea and America, existed difference, stating that culture has greater influence. Regarding previous studies, researches on preference in lateral attraction vary depending on gender, age, orthodontic knowledge, and culture.

In this study, lateral facial pictures with normal range of anteroposterior position of lips relative to the esthetic line via cephalometric analysis. Tendencies for preference on anteroposterior position of the lip were similar in all groups. Both male and female groups preferred lateral appearance with retrusive lips rather than protrusive lips, indicating no difference in preference on position of lips.

Recognition for facial esthetics can vary among professionals and patients, and this may influence diagnosis, treatment plan, and evaluation of treatment. Thus, studies were conducted regarding this matter. Romani et al. ${ }^{26)}$ reported that there is no difference in cognition of facial attraction among professionals and patients, and Peck and Peck ${ }^{27}$ said that the appearance preferred by public, as in a beauty contest, did not fulfill the standards for the professionals in their study. Prahl-Andersen et al. ${ }^{28)}$ and Bell et al. ${ }^{29)}$ also reported that there exists difference in preferences of public and professionals in facial attractiveness. Lines et al. ${ }^{30)}$ said that orthodontists generally prefer protrusive lips in their study on attractiveness of lateral appearance via silhouette with altered lips, nose, and jaws.

A comparison between group with at least a year of dental education and a public group was made in this study, considering that the preference for lateral 
attractiveness can be affected by dental knowledge and professionality according to previous studies. The result revealed that no difference was found in preferences of two groups. Both groups preferred retrusive lip appearance over protrusive lip in relation to the esthetic line. In professional group, a significant difference was found compared to the public group when lips were protruded for $2 \mathrm{~mm}$, obviously refusing protruded lateral appearance.

This study categorized appraisers, twenty to forty years of age, according to their gender and extent of professional knowledge. In all groups, preference for lip protrusion on lateral appearance in relation to the esthetic line was uniform, preferring retrusive lip position but refusing protrusive lip position. However, members of the professional group were mostly students with dentistry major, and this indicates that there might exist limitations in separating them from public group as they had no experience in orthodontic diagnosis and treatment. Following researches should conduct comparison of a group consisted of dentists who have experienced orthodontic diagnosis and treatment with a public group.

\section{Conclusion}

In this study, the preference for the extent of lip protrusion favored retrusive lips over protrusive lips regardless of dental knowledge or gender, and the professional group displayer more obvious tendency for unfavoring lateral appearance with protrusive lips.

\section{Acknowledgement}

This research was financially supported by the Ministry of Knowledge Economy (MKE), Korea Institute for Advancement of Technology (KIAT) and Honam Leading Industry Office through the Leading Industry Development for Economic Region.

\section{References}

1. Baldwin DC. Appearance and aesthetics in oral health. Community Dent Oral Epidemiol. 1980; 8: 244-56.

2. Sarver DM. The esthetic impact of orthodontics: planning treatment to meet patients' needs. J Am Dent Assoc. 1993; 124: 99-102.

3. Papel ID. Quantitative facial aesthetic evaluation with computer imaging. Facial Plast Surg. 1990; 7: 35-44.

4. Foster EJ. Profile preferences among diversified groups. Angle Orthod. 1973; 43: 34-40.

5. Czarnecki ST, Nanda RS, Currier GF. Perceptions of a balanced facial profile. Am J Orthod Dentofacial Orthop. 1993; 104: 180-7.

6. Ricketts RM. Esthetics, environment, and the law of lip relation. Am J Orthod. 1968; 54: 272-89.

7. Steiner CC. The use of cephalometrics as an aid to planning and assessing orthodontic treatment. Am J Orthod. 1960; 46: 721-35.

8. Burstone CJ. Lip posture and its significance in treatment planning. Am J Orthod. 1967; 53: 262-84.

9. Holdaway RA. A soft-tissue cephalometric analysis and its use in orthodontic treatment planning. Part I. Am J Orthod. 1983; 84: 1-28.

10. Hsu BS. Comparisons of the five analytic reference lines of the horizontal lip position: their consistency and sensitivity. Am J Orthod Dentofacial Orthop. 1993; 104: 355-60.

11. Farrow AL, Zarrinnia K, Azizi K. Bimaxillary protrusion in black Americans--an esthetic evaluation and the treatment considerations. Am J Orthod Dentofacial Orthop. 1993; 104: 240-50.

12. Mantzikos T. Esthetic soft tissue profile preferences among the Japanese population. Am J Orthod Dentofacial Orthop. 1998; 114: 1-7.

13. Song S, Choi IC. A study on the facial esthetic preferences among Korean youths: assessment of profile preferences. Korean J Orthod. 1992; 22: 881920.

14. Ko SJ, Kim HS, Kim YJ. The influence of gender 
or culture on determining esthetic facial profile. Korean J Orthod. 2001; 31: 301-9.

15. Kazanis K. An evaluation of lip attractiveness. Place unknwon: Saint Louis University; 2012.

16. Choi JG, Lee KS. A study of esthetic facial profile preference in Korean. Korean J Orthod. 2002;32:327-42.

17. Burcal RG, Laskin DM, Sperry TP. Recognition of profile change after simulated orthognathic surgery. J Oral Maxillofac Surg. 1987; 45: 666-70.

18. Faure JC, Rieffe C, Maltha JC. The influence of different facial components on facial aesthetics. Eur J Orthod. 2002; 24: 1-7.

19. Phillips C, Tulloch C, Dann C. Rating of facial attractiveness. Community Dent Oral Epidemiol. 1992; 20: 214-20.

20. Howells DJ, Shaw WC. The validity and reliability of ratings of dental and facial attractiveness for epidemiologic use. Am J Orthod. 1985; 88: 402-8.

21. Aitken RC. Measurement of feelings using visual analogue scales. Proc R Soc Med. 1969; 62: 989-93.

22. Soh J, Chew MT, Wong HB. A comparative assessment of the perception of Chinese facial profile esthetics. Am J Orthod Dentofacial Orthop. 2005; 127: 692-9.

23. Soh J, Chew MT, Wong HB. Professional assessment of facial profile attractiveness. Am J Orthod Dentofacial Orthop. 2005; 128: 201-5.
24. Nomura M, Motegi E, Hatch JP, Gakunga PT, Ng'ang'a PM, Rugh JD, Yamaguchi H. Esthetic preferences of European American, Hispanic American, Japanese, and African judges for softtissue profiles. Am J Orthod Dentofacial Orthop. 2009; 135(4 suppl): S87-95.

25. Kuroda S, Sugahara T, Takabatake S, Taketa H, Ando R, Takano-Yamamoto T. Influence of anteroposterior mandibular positions on facial attractiveness in Japanese adults. Am J Orthod Dentofacial Orthop. 2009; 135: 73-8.

26. Romani KL, Agahi F, Nanda R, Zernik JH. Evaluation of horizontal and vertical differences in facial profiles by orthodontists and lay people. Angle Orthod. 1993; 63: 175-82.

27. Peck H, Peck S. A concept of facial esthetics. Angle Orthod. 1970; 40: 284-318.

28. Prahl-Andersen B, Boersma H, van der Linden FP, Moore AW. Perceptions of dentofacial morphology by laypersons, general dentists, and orthodontists. J Am Dent Assoc. 1979; 98: 209-12.

29. Bell R, Kiyak HA, Joondeph DR, McNeill RW, Wallen TR. Perceptions of facial profile and their influence on the decision to undergo orthognathic surgery. Am J Orthod. 1985; 88: 323-32.

30. Lines PA, Lines RR, Lines CA. Profilemetrics and facial esthetics. Am J Orthod. 1978; 73: 648-57. 